\title{
Author Index, 6th International Psoriasis Symposium
}

Numbers refer to Abstract numbers

Abdel Rasik, M.M. 35 Abi-Rached, J. 24 Accardo, S. 17 Aiba,S. 55 Akimov,V.G. 27 Almanzor, J. 1 Altmeyer, P. 73 Amalyan, V.A. 62 Andrashko, V.U. 63 Arifov, S. 23 Austad, J. 76

Avrach,W. 68

Bachmann, H. 81 Badokin,V.V. 29,121 Badr,M.F. 34 Bamford, J.T.M. 30 Bardazzi,F. 114

Barnes, L. 12 Barone, A. 17 Beer,W. 61 Beijersbergen, R.L. 84,

96 Bernd,A. 31 Bezemer,A.C. 84,96 Bikowski, J. 32 Bjerring, P. 13 Blankush, J.L. 30

Bocharova, E.N. 71,72 Boehm,N. 41 Bos,J.D. 4,5,7,25,33,

116,122 Bossuyt, J.J.E. 116 Bossuyt, P.M.M. 7 Bräutigam, M. 74,100,

109 Brenner, S. 68,97 Brezzi, G. 103 Bright, R.D. 38 Brodecka, H. 43 Buckley, D. 20

Caputo,R. 103 Carducci, M. 79 Carlberg,C. 8 Castagnetta, L. 17 Cattaneo,A. 80

Cavicchini, S. 103 Charuwichitratana, S.

110 Chateau, D. 41 Chevret, S. 24 Christophers, E. 47,50,

81 Clark, A.R. 19,98 Cortés-Franco, R. 18 Cotterill, J.A. 57,58 Cubanova, A.A. 27 Cutolo,M. 17

Dang, M. 1 Dehdashti,D. 46 Delia Casa-Alberighi, O.

79 Didierjean, L. 9 Diffey,B.L. 107 Domínguez-Soto, L. 18 Dovzhanskaya, V. 36 Dovzhansky,

D. 37 Dovzhansky, I. 37 Dovzhansky, S. 36 Duarte, M. 60 Dubertret, L. 24

Eid,M.M. 34 Elder, J.T. 47 El-Shamy, S.I. 34,35 El-Sharabasy, M.M. 35 Eman, A.M.E. 34

Epinette, W.W. 1 Evain-Brion, D. 39

Farber, E.M. 38, 59, 87 Färber,L. 81,102 Farr,P.M. 107 Feeley, J. 12 Feldman,S.R. 19,98

Feldmann, R. 9 Fernandez, L. 18 Ferrario, E. 80 Fialla,R. 22 Fiebiger, M. 22 Finzi, A.F. 79, 80

Fisher, D.A. 120

Fleischer, A.B., Jr. 19 Födinger, D. 22 Fullerton, A. 40 Furth,R.van 84,96

Garber,A.T. 59 Gasparini, G. 103 Geiger, J.-M. 41 Gerbaud,P. 39 Gerschitz,J. 109 Gilleaudeau, P. 69 Giryes, H. 42 Giusti, M. 17 Glinska-Ferenz, M. 43 Glinski,W. 43 Glogau, R.G. 120 Goetinck, P.F. 51 Gomberg,M \. 71,72 Goncharenko, M.S. 44 Gottlieb, A. 69 Gottlieb, S. 69 Granata,O.M. 17 Griffin, E.I. 46 Griffiths, C.E.M. 45 Grossman, R.M. 24 Guadagnini, A. 15 Guidetti, M.S. 114 Gulliver, W.P. 59 Guo, Y. 86 Guzzo,C. 77

Halevy,S. 42 Hallmayer, J. 59 Halpern, A. 77 Harris, H.R. 78 Hartwig, R. 73 Hegemann, L. 48 Heller, D. 3 Heng,M.C.Y. 49 Heng,M.K. 49 Henneicke-von Zepelin,

H.-H. 81 Henseler, T. 47, 50 Hibino, T. 51 Highton,A. 52,53 Hilliges,M. 93 Höcher,J. 102

Hogan,M.P. 83 Holzmann,H. 31,54,60 Honeyman, J.F. 2 Hör, G. 54,60 Hori, Y. 14,82 Horikoshi, T. 82 Horiuchi, N. 55

Iizuka,M. 10 Inokuchi, S. 10, 11 Ippolito,F. 79 Ishibashi,Y. 82

Jablonska, S. 33 Jacobi,V. 60 Jeffes, E.W.B. 56 Jenisch, S. 47 Johansson, O. 93 Johnson, R. 69

Kaltwasser, J.P. 54,60 Kang,S. 45 Kassab,J.Y. 61 Kaur,I. 70 Kawakubo,Y. 10 Kiel,U. 101

King, L.E., Jr. 83 Kiss, I. 53

Klusmann, A. 54, 60 Kokelj,F. 15,16 Kolbenstvedt, A. 76 Kolyadenko,V.G. 62,

63, 64, 67 Korol,V.N. 62,64,65, 
66,67 Korol,Y.G. 66 Kovbasyuk, S.A. 64 Kraaz,W. 28 Kragballe,K. 13,68 Krueger,J. 69 Kühn, B. 47 Kumar, B. 70

Landau, M. 68,97 Lavaroni, G. 15 Layton,A.M. 57,58 Leder,R.O. 59

111

Leoni, L. 79

Lier, R.A.W. van 25

Lin,B. 86

Litus, A.I. 63

Liu,G. 1

Lu,D. 86

Lundh Rosell, B. 93

McCormick, A. 1 McCullough, J.L. 1,56 McPhee, M.E. 52, 53 Magaton Rizzi, G. 16 Maibach, H.I. 120 Mansbridge, J.N. 75 Marcusson, J.A. 93 Marshall, W. 59 Mashkilleison, A. 90

Mashkilleyson, A.L. 71,

72 Matthes,U. 73 Meffert,H. 74 Meinardi, M.M.H.M. 5,

122 Meingassner, J.G. 22 Meola,T. 78 Michaëlsson, G. 28 Miltenburg, A.M.M. 96 Mohareb,

R.W. 21 Möll,F. 3

Morhenn,V.B. 75,95 Morita,K. 83 Mørk,N.-J. 76 Morsches, B. 99,100,

102, 109 Moustafa, F.E. 35 Mowad,C. 77 Moy,J.A. 78 Mozzanica, N. 79, 80 Mrowietz, U. 81

Müller-Ruchholtz, W. 47

Nair,R,P. 47 Nakagawa,H. 82 Nakayama,J. 14,82 Nanney,L.B. 83 Nibbering, P.H. 84,96 Nolle, M. 31 Nurre,L.D. 19 Nylen,P. 69

Ohkido,M. 10,11 Okido,M. 82 Olisova,M.O. 27

Oliver,B. 98 Øster,H. 13 O’Toole,E. 12 Out,TA. 25 Ozawa,A. 10,11,82

Panizzon, R. 3 Pauluzzi, P. 16 Peluso,A.M. 114 Petry,G 109 Piacquadio, D.J. 115 Polnikorn,N. 110 Ponec, M. 96 Protsenko, T.V. 89 Putz,E. 22

Rajatanavin, N. 110 Rapp,S-R. 19 Rappersberger, K. 22 Raynaud, F. 39 Reboussin, D.M. 19

Rehder,M. 102 Renier,C.M. 30 Rezk,RA. 34,35 Rie, MA. de 25 Rogers, S. 20 Romanenko,

V.N. 111,

112 Rowland Payne, C. 61 Rubins, A. 33 Ruzicka, Th. 33

Sakuntabhai, A. 107 Samama, B. 41 Sanchez, L. 2 Sanchez, M.R. 78 Saurat,J.-H. 8,9

Schachtmeyster, I.Y. 72 Schlotzhauer, A. 1 Schmitt,E.C. 79 Schmitt,M. 54,60 Schopf, R.E. 99, 100 ,

101, 102, 108, 109 Schreiber, A. 38 Sela,J. 97 Seriolo,B. 17 Serup, J. 40 Shakhtmeister, I. 90

Shani,J. 97 Shephard,S. 3 Shevchenko, T.I. 6,88,

$89,91,94,105,106$,

111,112

Shimamura, K. 10,11 Shlopov,U.G. 6 Shlopov,V.G. 88,89,

91,94,105,106,111,

112 Shlopova, N.B. 106 Shteiman,S. 97 Siskin, S.B. 53 Slesarenko, N. 36 Smith, A. 61

Sobh,MA. 35 Sokolovsky, E.V. 26 Song,M.K. 49 Soter,NA. 78 Spellman, M.C. 115 Spuls,Ph.I.

7,116 Stinson, J. 12 Strumia, R. 104 Stütz,A. 22 Sugai,J. 10,11,82 Sukenik,S. 42 Sulli,A. 17

Sun,R.-z. 113 Sundberg,J. 83 Sundqvist, K.-G. 93 Suri,B. 77 Svistunov, I.V. 105 Synnestvedt, M. 77 Szarmach, H. 33

Tagami,H. 55,92 Takahashi,M. 82 Takahashi, T. 51 Takeda, A. 51 Talme,T. 93 Tamaoki,N.

10,11 Thami,G.P. 70 Theeu,M. 38 Theeuwes, M. 95 Thio,B. 84,96 Toll,L. 75 Tong,D. 22 
Tosti,M.S. 114 Trabattoni, D. 80 Trevisan, G. 16 Tur,E. 97 Turing, A. 108 Turing, T. 108 Tyson, M.E. 98

Ueyama,Y. 10,11 Urabe,A. 14

Valdés, P. 2

Van Everdingen, J.J.E. 7 Van Everdingen, L. 116 Varotti,C. 114 Vasilishin, A.A. 63 Vermeer, B.-J. 96 Villaggio,B. 17 Vladimirov, V. 90 Volos,L.I. 91 Voorhees, J.J. 47 Voss, J. 1 Voss, K. 1 Wald,E. 97 Waleh,N.S. 75 Wasik,P. 33 Weber, H. 99 Weinstein, G.D. 1,56 Weltman,H. 59

Welzel,D. 81 Werner, E. 60 Werner, R. 54 Westphal,E. 47 Wetterberg, L. 93 Wilkinson, D.I. 87 Witkamp, L. 4, 5, 7,25,

33,116,122 Wolff, K. 22,48 Woodworth,T. 69

Xue, Y. 86

Yereshchenko, Y.S. 44 Young, M.M. 85 Yudin,V.M. 64 Yue,T.-1. 113

Zachariae,H. 117,118,

119 Zachariae, R. 13 Zackheim, H.S. 120 Zalkiyev, R. 121 Zanolli,M,D. 98 Zheng, M.-r. 113 Zhou,D.-y. 113 Zöller,A. 60 Zomerdijk, T.P.L. 84,

96 Zonneveld, I. 33 Zonneveld, I.M. 4,25,

122

112

Author Index 